Anales del Seminario de Historia de la Filosofía ISSNe: 1988-2564

http://dx.doi.org/10.5209/ASHF.56104

\title{
¿Es el concepto platónico del amor intelectualista? Eros como impulso contemplativo y desiderativo en Platón
}

\author{
Manuel Cruz, Ortiz de Landázuri ${ }^{1}$
}

Recibido: 10/12/2015 / Aceptado: 29/12/2016

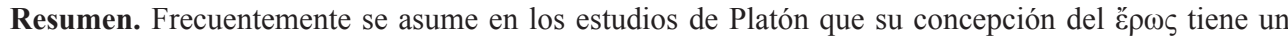
mero carácter instrumental respecto a la contemplación del bien, la belleza y la justicia; ha sido objeto de debate hasta qué punto los sentimientos y apetitos tienen importancia en su teoría del amor. Podría parecer que para Platón lo verdaderamente importante habría sido el conocimiento, y el amor, en todo caso, sólo entra en la vida porque supone un impulso para la contemplación. Frente a esta interpretación intelectualista del amor propongo una lectura de Platón (concretamente de algunos pasajes del Banquete, Fedro y República) que interprete el amor, en la línea de Charles Kahn, como una fuerza inscrita en lo más profundo del ser humano. Deseo, contemplación y acción no aparecen como conceptos contrarios, sino como elementos fundamentales de su ética.

Palabras clave: Amor; deseo; eros; intelectualismo; Platón.

\section{[en] Is the Platonic Concept of Love Intellectualistic? Eros as Contemplative and Desiderative Impulse in Plato}

\begin{abstract}
It is often assumed in Platonic studies that his conception of है $\rho \omega \varsigma$ is a mere instrumental desire for the contemplation of goodness, beauty and justice. It has been recently discussed how the feelings and desires are important in his theory of love. It might seem that for Plato knowledge is truly important, and love, in any case, only comes into life because is a boost for contemplation. Faced with this intellectualist interpretation of love I propose a reading of Plato (Symposium, Phaedrus and Republic) that understands love, in the line of Charles Kahn, as a force inscribed in the depths of the human being. Desire, contemplation and action do not appear as opposite concepts, but as key elements of his ethics.
\end{abstract}

KeyWords: Desire; eros; intellectualism; love; Plato.

Sumario. 1. Introducción. 2. La exposición de Diotima en el Banquete. 3. El amor aparente y el amor verdadero. 4. La purificación del amor en la República. 5. Conclusiones.

Cómo citar: Manuel Cruz, Ortiz de Landázuri (2017): “¿Es el concepto platónico del amor intelectualista? Eros como impulso contemplativo y desiderativo en Platón", en Anales del Seminario de Historia de la Filosofia 34 (2), 323-339.

1 Profesor ayudante doctor

Departamento de Filosofía

Universidad de Navarra.

mcruz@unav.es 


\section{Introducción}

Escribía Víctor Brochard a comienzos del siglo XX, a propósito de la noción plató-

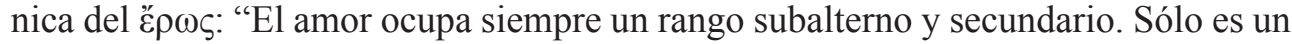
intermediario y un auxiliar. Su misión consiste en una propaganda moral y espiritual, permanece siempre por debajo de la verdadera ciencia"2. El pensador francés resumía así lo que buena parte de los estudios sobre Platón han presentado sobre su filosofía del amor. Platón sería un filósofo intelectualista, interesado en la contemplación especulativa, la argumentación y las ideas, y el amor sería una fuerza apetititva instrumental respecto a lo verdaderamente importante ${ }^{3}$. El amor podría interpretarse entonces como un motor hacia la verdad, un intermediario que desaparece una vez se llega a la contemplación de las ideas ${ }^{4}$; de este modo, el amor sería un deseo que desaparece en el mismo momento en que se consigue lo deseado.

En una época más reciente se ha ido desarrollando otra concepción del amor en clave intelectualista, en la línea de una discusión en torno a la posibilidad de amor interpersonal en Platón. Vlastos suscitó la cuestión de qué papel jugaba el amor interpersonal en el tratamiento del ě $\rho \omega \varsigma$ e interpretaba que no era más que un amor instrumental respecto al verdaderamente importante, el amor filosófico ${ }^{5}$. El amor sería en el fondo para Platón el impulso hacia el pleno conocimiento de la belleza intelectual y el amor a los particulares quedaría supeditado al amor intelectual.

Por otro lado, tampoco han faltado en los últimos años aproximaciones al Banquete de Platón y su teoría del épos en clave práctica, prescindiendo totalmente de referencias a la contemplación y el ascenso en niveles de conocimiento a través del amor. De este modo se ha estudiado el amor en Platón despojando cualquier referencia a la metafísica de las ideas y, consiguientemente, abandonando cualquier atisbo de intelectualismo ${ }^{6}$.

En las próximas páginas propongo argumentar que la concepción del amor como deseo de belleza en Platón supone una estructura desiderativa profunda en el ser humano que aspira a un objeto máximamente pleno para la contemplación humana. El amor no es un deseo más entre otros, sino un deseo permanente y total en el ser humano que busca algo máximamente pleno. De este modo quisiera aclarar la relación entre el amor, los deseos y la contemplación. Los deseos y apetitos sensibles son sólo los primeros pasos hacia el amor perfecto, pero esto tampoco quiere decir que la postura de Platón sea intelectualista. Parece posible leer su filosofía del amor como un intento de presentar una visión que va más allá de las opiniones sofistas y que

\footnotetext{
Brochard, V. “Sobre el Banquete de Platón”. Estudios sobre Sócrates y Platón, Losada, Buenos Aires, 1945, p. 68. También en esta línea escribía L. Robin: "El amor es un intermediario, pero un intermediario entre el mundo de los fenómenos y el de las Ideas: conduce en efecto al conocimiento de lo Bello en sí, del cual es amor, del mismo modo que de todo aquello que es inmutable, y dota precisamente al alma de un medio de salida, mediante la reminiscencia, de las conjeturas de la opinión que, como es sabido, son la región del alma caída”. RoBin, L. La théorie platonicienne de l'amour. Presses Universitaires de France, Paris, 1964, pp. 169-170.

4 Cfr. Robin, L. Platon. Phèdre. Belles Lettres, Paris, 1933, p. cxxxix.

5 Cfr. Vlastos. "The Individual as an Object of Love in Plato". Platonic Studies, Princeton University Press, Princeton, 1983, pp. 3-42.

6 Una en clave práctica se encuentra en Wedgwood, R. "Diotima's Eudaemonism: Intrinsic Value and Rational Motivation in Plato's Symposium”. Phronesis, 54, 2009, pp. 297-325. Wedgwood entiende la posesión de la belleza y el bien a la que aspira el amor como una relación adecuada respecto a un valor intrínseco. Parece subrayar así el aspecto práctico del amor como deseo de belleza, pero no considera la contemplación como forma de poseer auténticamente y unirse a la belleza, cuando este es un punto fundamental en toda la doctrina platónica del amor.
} 
trata de vincularla con la contemplación y la filosofía. Por eso mismo propongo una filosofía del amor a través de una lectura de tres pasajes importantes de sus diálogos medios: la exposición del discurso de Diotima en el Banquete, el papel del amor en

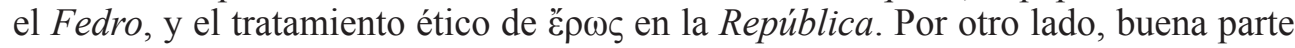
de la visión intelectualista del amor puede hundir sus raíces en una comprensión de Platón desde la teoría de las ideas en clave especulativa: si lo importante es para Platón la contemplación de la idea, todo lo demás (también el amor) resulta secundario. Sin embargo, ¿realmente son las ideas para Platón un objeto de pura contemplación intelectual sin que intervenga para nada la acción y el deseo? Por último, defenderé que detrás de esta concepción del amor no hay un proceso de sublimación de los deseos hacia el terreno intelectual, sino más bien una superación o búsqueda de algo máximamente perfecto.

\section{La exposición de Diotima en el Banquete}

Antes de entrar a analizar el amor en el Banquete conviene situar el tratamiento del ह̌ $\rho \omega \varsigma$ en una perspectiva adecuada. En primer lugar la palabra ह̌ $\rho \omega \varsigma$ resulta un término ambiguo que abarca desde el deseo sexual hasta el amor afectivo, e incluso el amor hacia los bienes materiales ${ }^{7}$. Por otro lado, hay que situar el tratamiento del ¿̌ $\rho \omega \varsigma$ en el contexto que Platón ha querido situarlo. ¿De qué hablan los comensales cuando encomian a ěp $\omega \varsigma$ ? El Banquete es un diálogo donde reina la ironía, la parodia, aunque también lo serio, y Platón presenta, a través de diversos personajes y discursos, el pensamiento sobre el amor de la aristocracia ateniense $e^{8}$. Son exposiciones en las que la poesía, la visión religiosa y la práctica cultural aparecen entremezcladas con el pensamiento filosófico ${ }^{9}$. Sólo cuando habla Sócrates con la enseñanza de Diotima parece que se llega a una noción nueva y purificada del amor: "El amor es, en

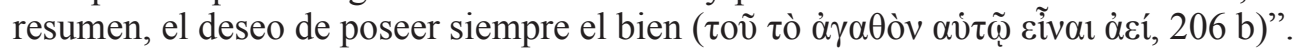
Aunque sería posible discutir qué papel juegan los discursos previos a Sócrates sobre la naturaleza del amor ${ }^{10}$, no cabe duda que la exposición que hace éste es la especulación más elaborada en el diálogo ${ }^{11}$. Las palabras de Sócrates suponen un discurso

7 Cfr. Chantraine, P. Dictionnaire etymologique de la langue grecque: histoire des mots. Klincksiek, Paris, 19681977 , p. 363.

8 Cfr. Diez, G. "Platons Symposion. Symbolbezüge und Symbolverständnis". Symbolon, 4, 1978, pp. 66-67.

9 Halperin sugiere que Platón muestra la aristocracia de la esplendorosa Atenas de algunos años antes y cómo su visión del amor y su forma de vida habrían llevado al traste la grandeza de la ciudad: "Plato invites the reader, in

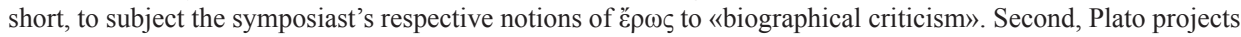
the speeches about है $\rho \omega \varsigma$ backwards to a period when Athenian power was at its height and all of the speakers were enjoying great personal prosperity. [...] Plato seems to locate a cause for the fall of Athens and for the ruin of its leading citizens in a failure of love, in the vicissitudes of a misguided है $\rho \omega \varsigma$ ". HaLPERIN, D. M. "Plato and the Erotics of Narrativity". Oxford Studies in Ancient Philosophy, Suppl. Vol. 1992, p. 100.

10 Cfr. Sier, K. Die Rede der Diotima. B. G. Teubner, Stuttgart und Leipzig, 1997, pp. XII-XVI.

11 C. Horn señala que es posible hablar de tres interpretaciones en la relación de los discursos del Banquete con la doctrina de Platón sobre el amor: en primer lugar la "interpretación clásica", según la cual la doctrina de Platón estaría contenida en el discurso de Sócrates sobre Diotima, y el resto de discursos serían exposiciones más o menos banales que preparan el camino a Sócrates; en segundo lugar se podría decir que Platón también habría expuesto parte de su doctrina del amor en los otros discursos; en tercer lugar Horn defiende que la doctrina del amor no está contenida del todo en ninguno de esos discursos ni en la suma de todos ellos. Cfr. Horn, C. "Enthält das Symposion Platons Theorie der Liebe?’. Platon. Symposion, C. Horn (ed.). Akademie Verlag, Berlin, 2012, pp. 1-16. En este estudio me inclino por seguir la interpretación clásica o, al menos, a centrar la atención en la enseñanza de Diotima, puesto que supone, sin duda alguna, la exposición más profunda que se encuentra en el 
filosófico que eleva el diálogo a otro plano de realidad ${ }^{12} \mathrm{y}$, por eso mismo, el análisis del amor en esta obra conviene centrarla en el discurso de Sócrates.

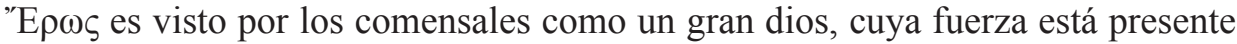
en toda la naturaleza e inspira sabiduría: es deseo, apetito y fuerza que arrastra. Sin

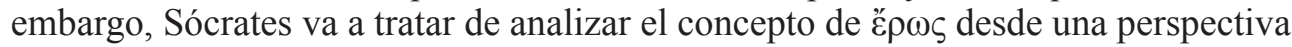
diferente: del deseo sexual pasa a tratar del deseo de felicidad, pero esto sólo se en-

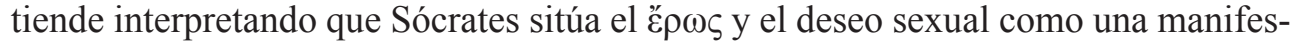
tación del deseo de felicidad ${ }^{13}$. Parece que todo el esfuerzo de Platón en el diálogo es

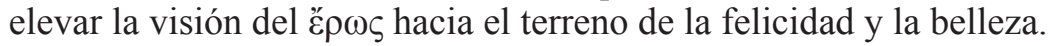

Aunque los discursos previso a Sócrates puedan aportar aspectos interesantes sobre el amor ${ }^{14}$, parece que Platón sitúa a Sócrates en un plano distinto mediante un relato en el que cuenta cómo Diotima le purificó de sus opiniones iniciales sobre la naturaleza del amor (201 e). Sin embargo, antes de comenzar su discurso Sócrates le plantea a Agatón que el amor es precisamente deseo de algo que no se tiene o, por lo menos, deseo de conservar en el futuro aquello que ya se posee: "También éste y cualquier otro que sienta deseo, desea lo que no tiene a su disposición y no está presente, lo que no posee, lo que él no es y de lo que está falto" (200 d-e). Se entiende entonces que el amor, aunque es un tipo de deseo, es un deseo peculiar, porque parece aspirar a poseer algo para siempre. Esta característica hace que el amor no desaparezca una vez se logra su objeto, sino que más bien sigue presente como una fuerza que anima a mantenerlo.

La exposición de Diotima posee una clara carga dramática ascendente. En primer lugar hay que purificar la noción de amor, entendiendo que es un deseo y, por lo tanto un intermediario, algo entre la plenitud y la pobreza ( $\mu \varepsilon \tau \alpha \xi \grave{v}, 202$ a), porque sólo se puede desear aquello de lo que se está falto. Por eso mismo el amor, siendo deseo de belleza y bien, no puede ser en sí mismo considerado ni bello ni bueno.

El siguiente paso que da Diotima es mostrar cómo este impulso a la belleza es, en realidad, un deseo de engendrar ( $\gamma \varepsilon v \varepsilon ́ \sigma \theta \alpha 1,204$ e) en lo bello. Pues el modo adecuado de perpetuar la posesión de la belleza es engendrar algo nuevo que participe de ella: el amor es deseo de poseer siempre lo bueno y bello (206 a). Sin embargo, todo el razonamiento hasta este punto no ha sido sino un preámbulo que conduce a los verdaderos misterios del amor.

"Quien hasta aquí haya sido instruido en las cosas del amor, tras haber contemplado las cosas bellas en ordenada y correcta sucesión, descubrirá de repen-

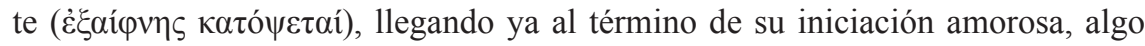

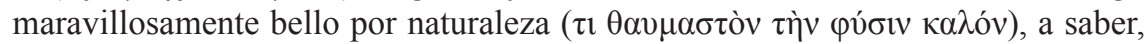
aquello mismo, Sócrates, por lo que precisamente se hicieron todos los esfuerzos

diálogo y, aunque ciertamente puede que no sea una elaboración completa, aporta una visión interesante sobre la relación de amor y belleza.

12 En opinión de Diez el Banquete representaría una estructura formal y simbólica perfecta. Los cinco primeros discursos sobre el amor parecen moverse en el plano de la opinión ( $\delta$ ó $\xi \alpha)$, mientras que la intervención de Sócrates (en dos partes) y la de Alcibíades están en el plano de la verdad ( $\dot{\alpha} \lambda \hat{\eta} \theta \varepsilon 1 \alpha)$; en el Banquete habría por tanto un equilibrio de composición perfecto incluso en el número de líneas que se dedican a la $\delta$ o $\xi \alpha$ y a la $\dot{\alpha} \lambda \eta \dot{\theta} \theta \varepsilon 1 \alpha$. Cfr. Diez, G. "Platons Symposion. Symbolbezüge und Symbolverständnis”. Symbolon, 4, 1978, p. 69.

13 Cfr. Sheffield, F. C. C. Plato's Symposium: The Ethics of Desire. University Press, Oxford, 2006, p. 78.

14 Sobre el papel de los discursos previos a Sócrates y los personajes cfr. Rowe, C. J. Plato. Symposium. Aris \& Phillips, Warminster, 1998, pp. 8-10. 
anteriores, que en primer lugar, existe siempre y ni nace ni perece, ni crece ni decrece; en segundo lugar, no es bello en un aspecto y feo en otro, ni unas veces bello y otras no, ni bello respecto a una cosa y feo respecto a otra, ni aquí bello y allí feo" (210 e-211 a).

El amor se presenta como deseo, pero todo deseo es suscitado por algo conocido o, por lo menos, experimentado. ¿Cómo se puede ansiar llegar a la belleza en sí cuando no se conoce de manera explícita? Porque esa belleza está presente en las bellezas particulares, que son las que despiertan el deseo hacia la contemplación de lo verdaderamente valioso. El camino del amor se presenta como ascenso hacia la contemplación pura de la belleza en sí misma, un camino arduo que supone un ejercicio ascético y cognoscitivo. A medida que se asciende en los niveles de belleza el amor será mejor y más profundo, puesto que las bellezas que se contemplan son cada

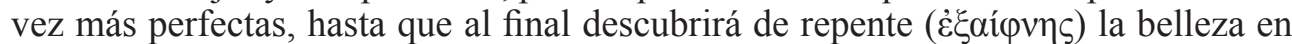
sí. El ascenso se lleva a cabo por medio del esfuerzo, pero el último paso es de contemplación directa, visión inteligible sin esfuerzo. Se puede hablar de cuatro pasos en este camino hacia la belleza: la contemplación de la belleza física individual, en la que se capta una hermosura que supera la particularidad del individuo; la apreciación de la belleza moral y la unidad existente entre todo lo bello; el impulso a encontrar la belleza en las verdades matemáticas; finalmente, la Belleza en sí misma por suerte de una intuición directa, sin mediaciones ${ }^{15}$.

Por tanto el amor se transforma a medida que el ser humano se purifica: se desea poseer siempre el bien y la belleza, y a través de la "elevación de la mirada" se descubre poco a poco que el bien y la belleza particular son tibias manifestaciones de algo más excelso e inmutable:

"Ni tampoco se le aparecerá esta belleza bajo la forma de un rostro ni de unas manos ni de cualquier otra cosa de las que participa un cuerpo, ni como un razo-

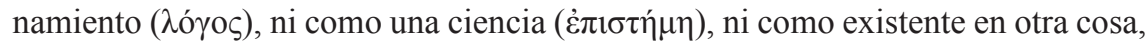
por ejemplo, en un ser vivo, en la tierra, en el cielo o en algún otro, sino la be-

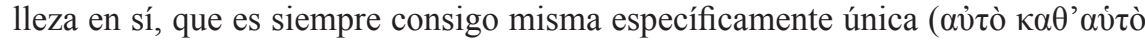

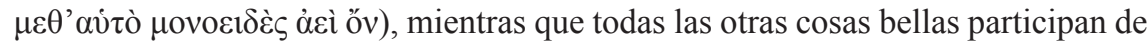

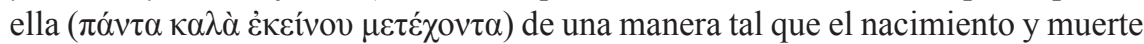
de éstas no le causa ni aumento ni disminución, ni le ocurre absolutamente nada" (211 a-b).

Esta belleza, que se encuentra como culmen de un proceso de ascenso, tiene dos ca-

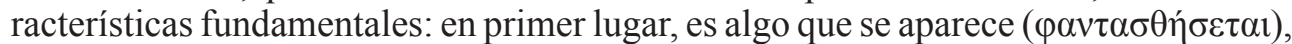

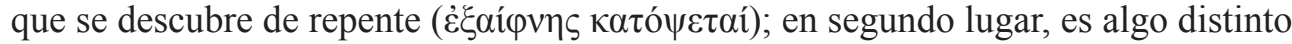

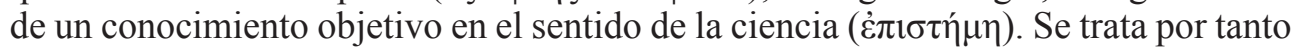
de un conocimiento directo de algo pleno e invariable.

Sin embargo, parece sugerir Platón que se trata de un ascenso estético, ético e intelectual: la belleza no sólo se aprecia en los cuerpos físicos o en las artes, sino también en el comportamiento ético y en la ciencia. El ser humano es capaz de ad-

15 Cfr. Cornford, F. M. “The Doctrine of Eros in Plato's Symposium”. Plato. A Collection of Critical Essays (vol. II). G. Vlastos (ed.), University of Notre Dame Press, Notre Dame, 1971, pp. 126-127. 
vertir la presencia de algo especial en todas aquellas cosas que llama "bellas", y la tarea del verdadero amante será el aspirar a una belleza pura que colme su anhelo profundo de bien:

"Ésta es justamente la manera correcta de acercarse a las cosas del amor o de ser conducido por otro: empezando por las cosas bellas de aquí y sirviéndose de ellas como de peldaños ir ascendiendo continuamente, en base a aquella belleza, de uno solo a dos y de dos a todos los cuerpos bellos y de los cuerpos bellos a las bellas normas de conducta, y de las normas de conducta a los bellos conocimientos ( $\tau \grave{\alpha}$ $\kappa \alpha \lambda \grave{\alpha} \mu \alpha \theta \eta \dot{\eta} \mu \alpha \tau \alpha)$, y partiendo de éstos terminar en aquel conocimiento que es co-

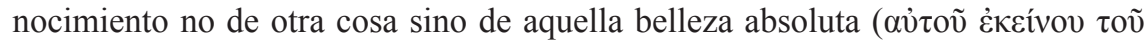

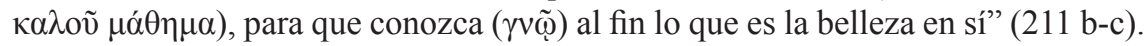

Por tanto el amor es aspiración a la belleza total, que se manifiesta primero en los cuerpos e imágenes sensibles y alcanza su cénit en la intelección o visión con los ojos del alma de aquello por lo cual todo lo demás se dice bello ${ }^{16}$. Este acto de conocimiento se sitúa más allá de los conocimientos objetivos, es decir, aquellas disciplinas que versan sobre objetos concretos y variados, y se centra en la belleza en sí misma, la cual no se identifica con ningún objeto concreto.

De este modo plantea Platón una nueva noción del amor purificado por la contemplación de la belleza en sí. El amor es bueno y noble cuando aspira a lo bueno y noble en sí, mientras que si se queda en el impulso a las bellezas corporales y sensibles arrastra al hombre a una vida infeliz. Sin embargo, en el Banquete no queda clara la posible armonía entre el apetito y la razón. El amor parece ser una fuerza irracional, aunque pueda aspirar a lo inteligible. Pero, ante todo, el amor para Platón no es un deseo puntual y concreto como pueden ser los deseos sensibles. El amor es un deseo profundo de inmortalidad, de bien, de belleza: el amor es el anhelo de perfección interior y exterior, de felicidad:

"Es necesario, según lo acordado, desear la inmortalidad (ả $\theta \alpha v \alpha \sigma i ́ \alpha \varsigma)$ junto con el bien, si realmente el amor tiene por objeto la perpetua posesión del bien ( $\tau$ õ

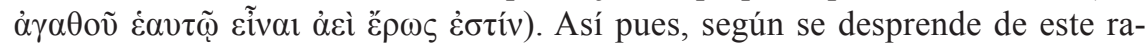
zonamiento, necesariamente el amor es también amor de la inmortalidad" (206 e-207 a).

Visto así el amor, ¿habrá que decir que la concepción de Platón en el Banquete intelectualista? ¿Sitúa el amor como mera fuerza o apetito instrumental de cara a la contemplación de la belleza? Es preciso preguntarse si para Platón esa contemplación de la belleza es sólo un puro ejercicio intelectual o más bien se trata de una captación directa que va incluso más allá del ejercicio ordinario del intelecto. Por otro lado, ese deseo de belleza va unido a un deseo de inmortalidad.

16 "The final act of knowledge is described as an immediate intuition in which there is no longer any process of thought. [...] Perhaps Plato's experience should be called metaphysical, rather than religious -a recognition of ultimate truth. On the other hand, it is not purely intellectual, but a conversion of every element in the soul by the last transfiguration of Eros". Cornford, F. M. "The Doctrine of Eros in Plato's Symposium". Plato. A Collection of Critical Essays (vol. II). G. Vlastos (ed.), University of Notre Dame Press, Notre Dame, 1971, p. 128. 
Respecto al pasaje central del discurso de Diotima en el Banquete se han dado dos líneas de interpretación que, o bien intentan entender el ascenso a la belleza mezclándolo con elementos del amor personal (sería una manera de evitar el intelectualismo $)^{17}$, o bien comprenden que el propósito de Platón es puramente intelectualista, sin que haya en su doctrina del amor espacio para el amor personal (sólo importaría el amor a las formas ${ }^{18}$. Dicho en forma de pregunta: ¿se debe entender el ascenso hacia la contemplación de la belleza en sí misma como una escalera en el que se abandonan los estadios anteriores (visión exclusivista) y, por lo tanto, el amor sólo aspira a contemplar la belleza en sí misma y nada más? O, por el contrario, ¿los estadios intermedios siguen siendo de interés para el amante que busca la belleza (visión inclusivista) ${ }^{19}$

La visión exclusivista parece estar fundamentada en la contemplación pura de la belleza, que parece conducir a prescindir de los individuos como objetos del amor ${ }^{20}$. Esta interpretación sería correcta desde una perspectiva intelectualista pura: si el único objeto capaz de saciar el amor es la contemplación de la belleza y a esta contemplación debo dirigir mi existencia, entonces mi trato con los demás seres humanos deberá ser únicamente instrumental, como medios en los que encuentro cierta belleza que me permite remontarme a una belleza mayor. Sin embargo, no hay razón en el Banquete que justifique una interpretación así. Se puede considerar que en el momento en que el amante descubre que su amado es una imagen de algo mejor, intentará contemplar eso mejor: no obstante, no por ello tendrá un trato instrumental en sus relaciones de amistad. Precisamente porque el amado sigue participando de la belleza tiene un valor intrínseco y puede seguir siendo amado mientras se busca una belleza mayor, en una esfera distinta de conocimiento ${ }^{21}$. Por otro lado, el ascenso hacia la belleza en el Banquete se describe siempre en un contexto educativo en el que la comunicación siempre aparece como $\mathrm{fin}^{22}$, de modo que el amor personal parece estar presente (o, por lo menos, no es algo que Platón rechace a favor de la belleza en sí misma). Platón apunta a la contemplación de la belleza en sí misma como la actividad que llena de felicidad y hace dichosa la vida, pero no hay en el discurso de Diotima una sola referencia que dé pie a entender que tal contemplación sea un impedimento para la amistad o que anule la contemplación del amigo. Es más, hay pasajes que dan pie a entender la necesidad de la auténtica amistad en la contemplación de la belleza, como por ejemplo cuando se describe el tipo de ayuda que Sócrates le intenta brindar a Alcibíades (218 d-e).

17 Cfr. Pater, W. Plato and Platonism. MacMillan, London, 1893, p. 170.

18 Moravcsik defiende así una interpretación "exclusivista" del ascenso: a medida que el amante asciende en los objetos de amor, los objetos anteriores son desechados. Cfr. MoravcsiK, J. M. E. "Reason and Eros in the Ascent Passage of the Symposium". Essays in Ancient Greek Philosophy. J. P. Anton y G. L. Kustas (eds.), Albany, 1971, p. 293.

19 Una buena exposición de ambas interpretaciones se encuentra en PRICE, A. W. Love and Friendship in Plato and Aristotle. Clarendon, Oxford, 1991, pp. 43-45.

20 Cfr. Vhastos. "The Individual as an Object of Love in Plato". Platonic Studies, Princeton University Press, Princeton, 1983, pp. 3-42.

21 Cfr. Lear, G. R., Happy Lives and the Highest Good. University Press, Princeton, 2004, p. 219.

22 "If the ascent passage has standardly been read as describing a discarding process of persons for the sake of Forms, that is, if I am right, the result of two connected mistakes (whose effect is only slightly mitigated by an inclusive reading): confusing the loved one's role as an object of contemplation (in which he is soon largely superseded) with his role as a recipient of thought, and taking the passage out of context". Price, A. W. Love and Friendship in Plato and Aristotle. Clarendon, Oxford, 1991, p. 49. 
Gran parte del problema en la interpretación intelectualista reside en situar el tratamiento del Banquete dentro de una teoría general del amor en la que Platón distinguiría netamente los conceptos: la philía, tratada en el Lisis y referida a la utilidad, y el čp $\rho \varsigma$, referido a la belleza y más intenso que la philía ${ }^{23}$. Sin embargo, es más que cuestionable que Platón tuviese en mente tal teoría general del amor. El amor es en el Banquete deseo de plenitud. Esa plenitud se alcanza, sin duda alguna, en la contemplación pura de la belleza. Ahora bien, no hay razón para admitir que Platón niegue el amor como un proceso inclusivo en el que se asciende hacia la belleza total mientras se contemplan las bellezas particulares. Es más, parece sugerir Platón que para lograr la belleza total hace falta la práctica $(\mu \varepsilon \lambda \varepsilon \tau \tilde{\alpha} \nu)$, de modo que se renueve

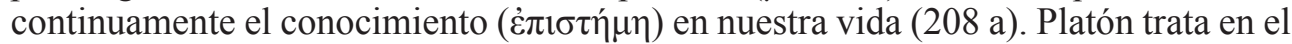
Banquete acerca del amor como ह̌ $\rho \omega \varsigma$, es decir, como deseo, y elabora una propuesta metafísica y psicológica de hacia qué apunta ese deseo (la belleza); no encontramos en el Banquete un desarrollo del amor como $\varphi \imath \lambda i ́ a$, es decir, como amor interpersonal (tal como hace Aristóteles en el libro IX de la Ética a Nicómaco). En otras palabras, Platón se preocupa de responder a la pregunta: ¿qué es el amor como deseo? A lo cual contesta: es aspiración a contemplar una belleza total. Pero sería inadecuado leer el Banquete pensando que Platón está tratando de responder a la pregunta ia quién y cómo tengo que amar?, puesto que no es eso lo que está analizando.

Por otro lado, cabría interpretar la Belleza del mismo modo en que aparece la contemplación del Bien en la República: el Bien no se observa de manera directa (porque ciega el entendimiento, sino que es aquello que dota de luz al resto de Ideas. La Belleza es aquello que dota de belleza al resto de cosas, permitiendo su contemplación. Si esto es así, entonces la belleza no es un mero objeto de contemplación individual, sino aquello que permite la contemplación de las bellezas particulares.

\section{El amor aparente y el amor verdadero}

El segundo tratamiento sobre el amor se encuentra en el Fedro. Resulta especialmente interesante este diálogo porque complementa la visión del amor del Banquete, poniendo en conexión el amor con los deseos del alma. Sin embargo, aquí el proceso es inverso al Banquete: si en aquel diálogo los comensales alababan las maravillas

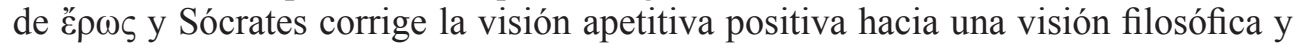
profunda, en el Fedro es el mismo Sócrates quien empieza despreciando el amor, por ser un mero apetito irracional, para luego corregir su propia visión e insertarla en el camino contemplativo del filósofo. En este caso los discursos sobre el amor se llevan a cabo desde un juego retórico con Fedro en el que se discute si es mejor que el amado ame realmente o no a su amante. En la primera intervención de Sócrates el amor es descrito como una fuerza dominadora, capaz de arrastrar al ser humano y encadenarle al mundo de las apariencias. El amor (ó ” E $\rho \omega \varsigma)$ pertenece al ámbito de los deseos que no tienen control racional (238 b-c) y que esclavizan al amante

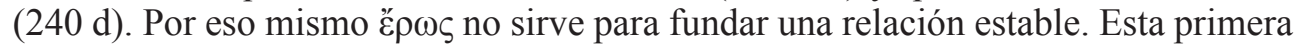
exposición del amor resulta enormemente negativa porque Sócrates se refiere a las formas de amor más sensibles y vinculadas con la parte irracional del ser humano. El amor así aparece unido, casi identificado, con la $\dot{\pi} \pi \theta v \mu i ́ \alpha$, fuerza ( $\dot{\rho} \omega \dot{\mu \eta})$ que

23 Cfr. Sheffield, F. C. C. Plato's Symposium: The Ethics of Desire. University Press, Oxford, 2006, p. 156. 
arrastra hacia el esplendor de los cuerpos y ciega a quien se ve por ella dominado

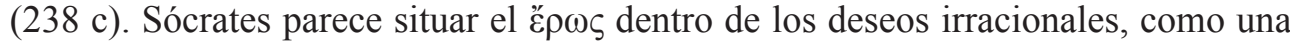
pasión e impulso incontrolado hacia lo bello: fuerza victoriosa $(\dot{\rho} \omega ́ \mu \eta)^{24}$. El amor no es más que un deseo sensible que permanece siempre insatisfecho y, por lo tanto, es la mayor fuente de desdichas.

Si el Fedro terminase aquí parecería que Platón destierra el amor de su filosofía y, sin embargo, esta primera exposición no es más que una introducción, pues Sócrates admite que el primer discurso acerca del amor ha sido excesivamente negativo y se propone emprender una exposición en clave alegórica. El amor es más que غ̇лı $\theta v \mu i ́ \alpha$ o, al menos, puede elevarse más allá de los apetitos, porque es el motor que mueve hacia la belleza. En el mito del carro alado ह̌ $\omega \varsigma$ no va a ser una fuerza irracional, ni se va a identificar con el deseo sensible, sino que se presenta como un deseo que

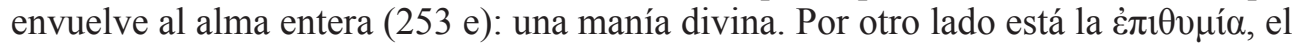
caballo negro, que tiende a rebelarse mientras el $\theta v \mu o ́ s$, caballo blanco, tiende a la obediencia. El amor, así pues, es ahora la fuerza o el impulso más profundo del hombre, que encuentra su objeto adecuado cuando el carro, liderado por la razón, avanza con orden ${ }^{25}$. Por eso ofrece Platón una descripción de las dos posibles actitudes del ser humano frente a la belleza:

"El que ya no es novicio o se ha corrompido, no se deja llevar, con presteza, de

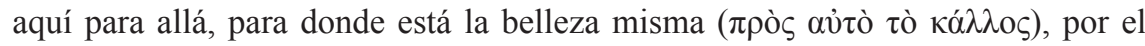
hecho de mirar lo que aquí tiene tal nombre, de forma que, al contemplarla, no siente estremecimiento alguno, sino que, dado al placer ( $\dot{\eta} \delta o v n ̃ ~ \pi \alpha \rho \alpha \delta o v ̀ \varsigma)$, pretende como un cuadrúpedo cubrir y hacer hijos, y muy versado ya en sus excesos,

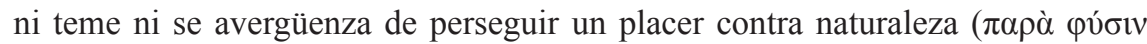
ทंठovìv)" (250 e-251 a).

De este modo describe la situación de la persona que se deja arrastrar por el placer sensible: esa persona ve los cuerpos bellos, pero no es capaz de contemplar, sino que persigue la satisfacción de sus apetitos. Tal actitud supone una perversión de la belleza, ya que no se busca contemplarla, sino acapararla y disfrutarla de manera mezquina. Pero a esta actitud Platón contrapone otra posibilidad:

"Sin embargo, aquel cuya iniciación es todavía reciente, el que contempló mucho de las de entonces, cuando ve un rostro de forma divina, o entrevé, en el cuerpo, una idea que imita a la belleza, se estremece primero, y le sobreviene algo de los temores de antaño y, después, lo venera, al mirarlo, como a un dios" (251 a).

En este caso se despierta el amor hacia el otro, que es un impulso hacia la belleza misma a través de la belleza particular del amado. Aquí se conecta el impulso a la belleza con la doctrina de la reminiscencia: el anhelo hacia la contemplación del ser (en esto consiste, en definitiva, el amor) se produce por una contemplación previa de las ideas. Por eso, al ver algo bello tiene lugar un recuerdo que provoca el deseo,

Cfr. Hackforth, R. Plato's Phaedrus. University Press, Cambridge, 1952, pp. 38-42.

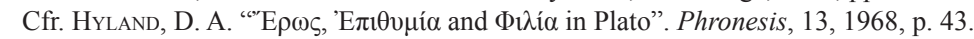


pero ese anhelo sólo logrará su plena satisfacción en la contemplación del ser, pues

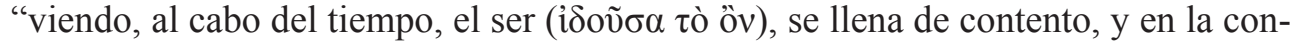
templación de la verdad $(\theta \varepsilon \omega \rho o \tilde{\sigma} \sigma \alpha \tau \dot{\alpha} \lambda \eta \theta \tilde{\eta})$ encuentra su alimento y bienestar" (247 d). De este modo Platón va a dar con una nueva noción del amor positiva, que es preciso purificar de los apetitos sensibles.

"Si vence la mejor parte de la mente, que conduce a una vida ordenada y a la filosofía, transcurre la existencia en felicidad y concordia, dueños de sí mismos

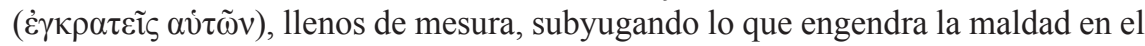
alma, y dejando en libertad a aquello en lo que lo excelente habita ( $\dot{\varepsilon} \lambda \varepsilon v \theta \varepsilon \rho \omega ́ \sigma \alpha \nu \tau \varepsilon \varsigma$ $\delta \dot{\varepsilon} \tilde{\omega} \alpha \dot{\alpha} \rho \varepsilon \tau \eta ́) ”(256 \mathrm{a}-\mathrm{b})$.

Por lo tanto el amor no es malo, pero tiene que ser purificado y guiado mediante el imperio de la razón. Sólo en la medida en que uno tiene dominio sobre los apetitos (uno es "dueño de sí") es cuando puede desarrollar libremente su parte más excelente del alma. El amor verdadero no es sólo una disposición amistosa, sino una pasión que absorbe toda la naturaleza humana en su complejidad y que lleva a salir de sí mismo ${ }^{26}$. El avance hacia el ser y la belleza en sí misma se presenta como una situación de equilibrio racional. El carro alado sólo se remonta a lo inteligible cuando se atempera la actitud del caballo negro, la $\dot{\varepsilon} \pi \imath \theta v \mu i ́ \alpha$, por orden del auriga, la inteligencia. Además, la fuerza que pone inicio a la marcha no es otra que el amor (č $\rho \omega \varsigma)$, como una manía divina inscrita en lo más profundo del hombre.

"E $\rho \omega \varsigma$ es un tipo de deseo, pero distinto de la $\dot{\pi} \pi \imath v \mu$ í $\alpha$ y del $\beta$ ov́ $\lambda \varepsilon \sigma \theta \alpha 1$. En buena medida parece que el esfuerzo de Platón en el Banquete y el Fedro es mostrar que

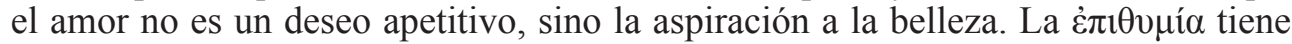
como objeto la satisfacción sensible inmediata, el placer corporal, mientras que ěp $\omega \varsigma$ tiene como objeto la belleza, la cual se presenta al principio al ser humano de manera corporal y sensible (acompañado de $\dot{\varepsilon} \pi \imath v \mu i ́ \alpha$ ), aunque luego pueda ser captada de

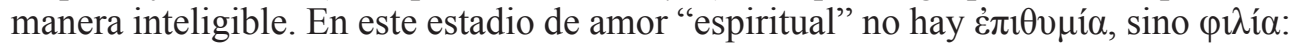

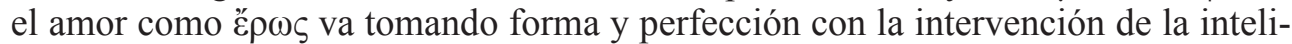
gencia y se vuelve amor al saber ${ }^{27}$. Pero aquí no hay sublimación (sustitución de lo apetitivo-sensible por lo intelectual), sino elevación: el amor aspira a algo pleno que no se encuentra en la región sensible, en las meras apariencias ${ }^{28}$. No se trata de que el amor sensible se reprima y redirija a otros aspectos de la vida (artísticos, intelectuales), sino que el amor, de suyo, aspira algo pleno y total, y necesita la elevación total del ser humano ${ }^{29}$.

\footnotetext{
Cfr. Thompson, W. H. The Phaedrus of Plato. Whittaker \& Co., London, 1868, p. 162.

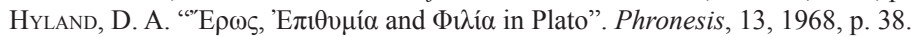

Cfr. Rowe, C. J. Plato. Symposium. Aris \& Phillips, Warminster, 1998, p. 7.

"The self-moving energy of the human soul resides properly in the highest part, the immortal nature. It does not arise from beneath, but rather sinks from above when the spirit is ensnared in the flesh. So, when the energy is withdrawn from the lower channels, it is gathered upo into its original source. This is indeed a conversion or transfiguration; but not a sublimation of desire that has hitherto existed only in the lower forms". CORNFORD, F. M. "The Doctrine of Eros in Plato's Symposium". Plato. A Collection of Critical Essays (vol. II). G. Vlastos (ed.), University of Notre Dame Press, Notre Dame, 1971, p. 129.
} 
Habría que preguntarse entonces si la exposición del amor en el Fedro proporciona una visión menos intelectualista que la del Banquete: al poner en conexión el amor con la tripartición del alma, parecería que el impuso amoroso abarca los aspectos sensibles y pasionales. Sin embargo, una interpretación así sólo podría hacerse partiendo de la base de que la exposición en el Banquete es intelectualista. Ciertamente en algunos momentos del Fedro da la impresión de que Platón otorga cierta importancia al elemento apetitivo en el amor, e incluso que es necesaria una respuesta sensible o sexual para que el है $\rho \omega \varsigma$ se desarrolle convenientemente pero no se debe perder de vista que la belleza sensible es sólo el comienzo, la chispa que despierta el recuerdo de lo verdaderamente real, y por lo tanto el primer escalón en el ascenso ${ }^{30}$. En realidad son más las continuidades entre el Banquete y el Fedro que las disimilitudes, y tampoco parece que en el Fedro Platón presente la respuesta apetitiva y sensible del amor como una condición necesaria: más bien lo sorprendente es que conecta el amor con un impulso que busca el descanso en la contemplación de las formas, verdadero alimento del alma ${ }^{31}$. La belleza no se alcanza con una respuesta apetitiva sino con una actitud contemplativa de elevación; la observación sensible de belleza es el camino hacia lo inteligible y puro $^{32}$.

Por último hay que preguntarse qué papel juegan las relaciones eróticas en la doctrina del amor del Fedro. Algunos pasajes del mito del carro alado y la relación entre amantes podrían dar pie a entender que el elemento apetitivo y pasional en las relaciones eróticas de larga duración entre individuos son algo necesario en la doctrina platónica del amor ${ }^{33}$. Sin embargo, hay que tener en cuenta que Platón dice que lo mejor es una relación de amor en la que no venza el apetito sensible, para poder ascender hacia las formas (256 a-b); es decir, que el amor mejor y deseable es aquel en que hay una relación entre el amante y el amado que supera lo apetitivo y sensible. La relación de amante y amado debe llevar a descubrir lo bueno y racional, lo cual sólo es posible en la medida en que se domestican los apetitos ${ }^{34}$.

\section{La purificación del amor en la República}

Se ha visto cómo en el Banquete el amor ha sido descrito primeramente por los comensales como una fuerza irracional que, en opinión de algunos, puede traer ciertos beneficios pero, posteriormente, Platón muestra a través de Sócrates que el amor

30 Cfr. Sheffield, F. “Eros Before and After Tripartition”. Plato and the Divided Self. R. Barney, T. Brennan, C. Brittain (eds.), Cambridge University Press, Cambridge, 2012, pp. 230-231.

31 Cfr. Sheffeld, F. "Eros Before and After Tripartition". Plato and the Divided Self. R. Barney, T. Brennan, C. Brittain (eds.), Cambridge University Press, Cambridge, 2012, p. 232.

32 La visión de la belleza corporal es camino hacia la contemplación de las ideas. Cfr. Grassi, E. Die Theorie des Schönen in der Antike. DuMont, Köln, 1980, p. 113. Sin embargo, eso no qui ere decir que el camino de la belleza necesite de la satisfacción de apetitos básicos, sino que a través de la contemplación de las bellezas particulares se despierta la inteligencia hacia la belleza en sí misma.

33 Cfr. Nussbaum, M., The Fragility of Goodness. Cambridge University Press, Cambridge, 1986, p. 201.

34 Buena parte de la interpretación de Nussbaum ha sido criticada por Rowe, al afirmar que precisamente el núcleo del mito del Fedro estriba en la excelencia de la vida racional entre los amantes que lleva a la contemplación de la verdad, y eso supone moderar los apetitos: "El descubrimiento hecho por el amante y su amado es exactamente la racionalidad que comparten con los dioses, y en la posibilidad de usar esto para domesticar los elementos irracionales. La diferencia de posición representada en otros diálogos es que se reconoce ahora con firmeza que estos elementos irracionales están siempre con nosotros". Rowe, C., "Philosophy, Love and Madness". The Person and the Human Mind. C. Gill (ed.), Clarendon Press, Oxford, 1990, p. 240. 
puede ser elevado hasta la contemplación de la belleza en sí. En el Fedro el amor aparece asociado en un primer momento a los apetitos sensibles, pero después resulta ser también una ayuda, vinculada al recuerdo, para la contemplación del ser. En la República Platón quiere depurar el amor, incorporarlo a su filosofía sin tener que desterrarlo al ámbito de los vicios ${ }^{35}$. El amor para eso tendrá que encontrar un objeto adecuado. De suyo tiende a las bellezas particulares, al gozo de los cuerpos, pero es preciso elevarlo hacia la belleza en sí, superior a todas las demás, que se alcanza mediante la contemplación intelectual. El verdadero amor es aquel orientado hacia lo realmente real, y eso sólo se consigue huyendo de las apariencias, desterrando los apetitos que impiden la auténtica contemplación. Sólo entonces el amor está purificado. En la República desarrolla Platón una exposición de la purificación del amor en consonancia con su gnoseología (apariencia sensible-realidad inteligible), donde distingue entre el placer del amor sexual y el amor puro y limpio:

“-¿Puedes citarme algún otro placer mayor ni más vivo que el placer venéreo

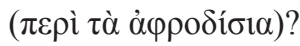

-No lo hay -respondió-, ni ninguno tampoco más parecido a la locura ( $\mu \alpha v i \kappa \omega \tau \varepsilon \dot{\rho} \rho \alpha)$.

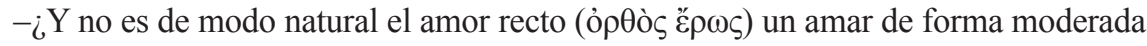

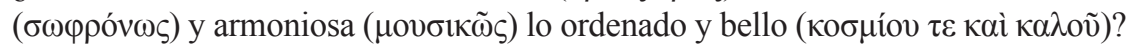
-Efectivamente -respondió.

-Entonces, ¿no hay que mezclar con el amor recto nada relacionado con la locura o la incontinencia?

-No, por Zeus -convino-, no se debe mezclar" (403 a).

El amor, por tanto, no es algo malo ni meramente apetitivo-sensible, sino que apunta a algo más alto, la belleza y bondad en sí. Ahora bien, será necesario purificar los apetitos y despejar la desmesura del verdadero amor ${ }^{36}$. Curiosamente aparece la música en la República como camino hacia el amor de la belleza: "Es preciso que la

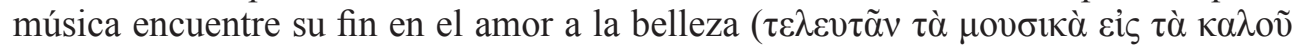

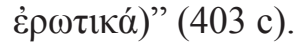

El conocimiento de lo bello en sí es un proceso de ascenso desde la belleza particular a la belleza en sí misma, y es el final del proceso una actividad contemplativa,

35 A menudo se ha discutido si Platón puede haber hecho algunas concesiones a la pederastia como práctica beneficiosa de cara al aprendizaje. A este respecto la postura de Effe resulta iluminadora: "Die theoretische Einstellung Platons zur Päderastie ist einheitlich und konsistent; der einzige Unterschied besteht Darin, dass mal die Kritik an der verbreiteten sexuellen Praktiken (Politeia, Nomoi), mal das philosophische Konzept einer entsexualisierten Homoerotik im Mittelpunkt des Interesses steht (Symposion, Phaidros): Die körperlichen, an sexuellen Lustgewinn orienterten Implikationen der Päderastie (wie sie die Zeitgenössische Geschellschaft innerhalb bestimmter Grenzen durchaus tolerierte) werden rundweg abgelehnt und als widernatürlich gebrandmarkt; und es wird, gleichsam als positive Kehrseite der Medaille, eine Theoire entworfen, welche die päderastische Praxis radikal entsexualisiert, bis zur Unkenntlichkeit ins Geistige sublimiert und aus dem gleichgeschlechtichen Eros einen priviligierten Weg der àvó $\mu v \eta \sigma ı \varsigma$ und einen Drang zu philosophischer Erkenntnis werden lässt". EFFE, B. "Platon und die Päderastie. Phaidros 256 b-d und die platonische Eros-Theorie". Platon verstehen. M. van Ackeren (ed.), Wissenschaftliche Buchgesellschaft, Darmstad, 2004, pp. 141-142. La aprobación de la pederastia en algunos de los discursos del Banquete hay que leerla en clave irónica respecto a las prácticas de la alta sociedad de su tiempo: tales discursos se sitúan entre la broma y la parodia de los personajes que los recitan. El discurso de Sócrates apunta a una superación de ese amor en un nivel espiritual.

36 Cfr. PIEPER, J. Begeisterung und göttlicher Wahnsinn. Kösel, München, 1962, pp. 118-119. 
y por eso la más digna de ser vivida ${ }^{37}$. Sin embargo Platón nos dice más acerca de lo que no es la belleza en sí misma que acerca de lo que es ${ }^{38}$, y de este modo parece que la noción de belleza en sí misma se acerca en gran medida a la idea de bien en la República, puesto que parece que es algo que no se puede mostrar, sino únicamente indicar el camino para su contemplación, puesto que no es esencia, sino algo que se

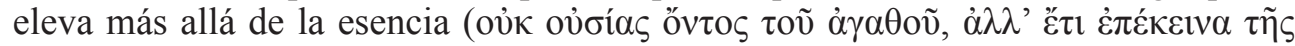
ov̉oías, 509 b). Esa contemplación del bien es además lo más hermoso: "Hablas de una belleza extraordinaria, puesto que produce la ciencia y la verdad, y además está

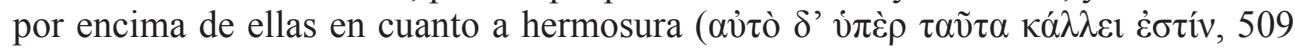
a)". Ahora bien, ¿se identifican el bien y la belleza en el Banquete? Parece que sí, puesto que el ascenso hacia ambas ideas es idéntico: se trata de un proceso de esclarecimiento de algo inmutable, estable y perfecto que va más allá de lo sensible y que es incomunicable por estar también más allá de todo razonamiento: lo que dota de sentido a la realidad y a las mismas ciencias no puede ser abarcado por ningún discurso. Se trata por tanto de la visión directa de una misma idea, aunque quizás bajo diversos aspectos ${ }^{39}$.

Para dilucidar si la concepción platónica del amor es intelectualista se hace preciso examinar la relación que guarda el amor con los tipos de deseos según la tripartición del alma en la República. Ciertamente el discurso de Diotima en el Banquete pivota sobre la transformación del deseo corporal -ligado a los apetitos (las bellezas particulares)- en un deseo de la belleza en sí, inteligible, mediante un ascenso purgativo en el que no se anulan los deseos anteriores, sino que se superan. ¿Es posible conjugar esta doctrina del amor con la distinción de deseos en la República? Evidentemente hay muchos puntos de convergencia en ambos diálogos: especialmente remarcable es la similitud entre la ascensión del prisionero de la caverna hacia la idea de bien y el ascenso del amante hacia la belleza en sí misma, pero, como ha señalado Charles Kahn ${ }^{40}$, parece necesario explicar cómo puede ser compatible la doctrina del amor del Banquete con la tripartición de los deseos. Dicho de modo más explícito: ¿cómo puede el deseo corporal ser transformado en amor a la Belleza en sí misma (o deseo del bien en sí)? No se trata de una cuestión menor, pues en definitiva hay que

37 Cfr. Banquete, $211 \mathrm{~d}$.

38 "In this famous account of the insight into beauty itself, we learn not a single thing discursively about what beauty itself is. We learn only -and this is true again and again in the various accounts of forms throughout the dialogues-a generic account of the nature of formal structure". HyLAnd, D. A. Plato and the Question of Beauty. Indiana University Press, Bloomington/Indianapolis, 2008, p. 57.

39 Según Sier hay una diferencia de aspecto entre el bien y la belleza en cuanto que la presencia de la belleza parece preceder al logro del fin (el bien): "Die Mehrzahl der Interpreten nimmt an, dass schön und gut funktional äquivalent stehen: mit $\tau \tilde{\omega} \nu \kappa \alpha \lambda \tilde{\omega} \nu$ (204 d 5) und $\tau \tilde{\omega} \nu \alpha \dot{\alpha} \alpha \theta \tilde{\omega} v$ (e 3) seien beidemal die Objekte des Eros gemeint; ein Unterschied bestehe nur im Aspekt, insofern das Momet des Nützlichen und der Eudaimonie Dienlichen eher mit dem Guten als mit dem Schönen assoziiert ist; das von Diotima vorgeschlagene $\mu \varepsilon \tau \alpha \beta$ o $\lambda \varepsilon \tilde{v} v$ des Ausdrucks wäre dann bloß als Verständnishilfe für Sokrates gedacht. [...] Das menschliche Telos ist das Gute, das heisst die Eudaimonie (205 d 1-206 a 1), genauer die «immerwährende Gegenwart des Guten für das Individuum» ( $\tau$ ò

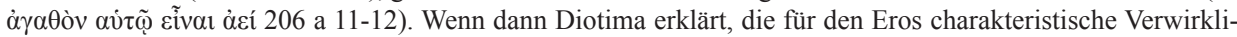
chung dieses Ziel sei das «Hervorbringen im Schönen» (206 b 1-8), so kann dies, unabhänging davon, was hier mit dem Eros gemeint ist, nur bedeuten, dass die Gegenwart des $\kappa \alpha \lambda$ óv dem Erreichen des Telos vorausgeht". SiER, K. Die Rede der Diotima. B. G. Teubner, Stuttgart und Leipzig, 1997, p. 98. Su postura interpreta el bien y la belleza desde el punto de vista de la felicidad y, en ese sentido, podría decirse que la belleza es camino hacia el bien; sin embargo, desde el punto de vista gnoseológico, parece que Platón se está refiriendo al mismo acto de contemplación.

40 Cfr. Kahn, C. "Plato's Theory of Desire". Review of Metaphysics, 41, 1987, pp. 95-96. 
dar respuesta a cómo es posible la purificación psicológica, interior, en el plano de los deseos, de cara a la contemplación de las ideas.

La propuesta de Kahn consiste en situar el Ěp $\rho \varsigma$ en un plano por detrás de los deseos de cada parte del alma. El amor no es un deseo más entre otros, tampoco pertenece exclusivamente a una parte del alma, sino que es un deseo profundo del ser humano que aspira a lograr la belleza y felicidad. Ese deseo profundo se manifiesta primero en el ámbito sensible, pero también en el pasional y por último en el

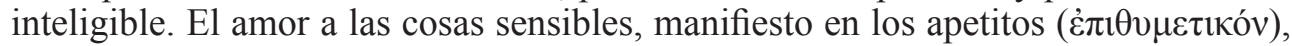
sería susceptible de ser elevado en amor a la belleza en sí misma, ámbito propio de lo

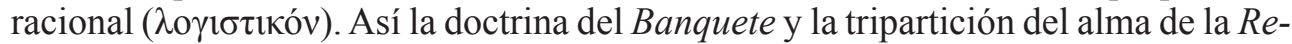
pública se mostrarían compatibles y complementarias: "Al igual que la conversión

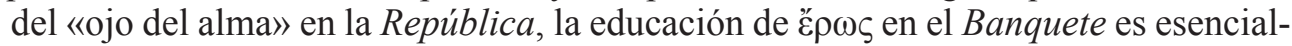
mente una empresa intelectual, la liberación del deseo racional de las ataduras de un objeto inadecuado y su redirección hacia su propio fin, «el conocimiento verdadero» que es conocimiento de la belleza en sí misma (211 c 7). El Banquete muestra claramente lo que está sólo parcialmente indicado en la descripción de reorientación en la República: que el proceso de alumbramiento de la razón es al mismo tiempo un proceso de reeducación de los deseos"41.

Es precisamente en este punto -en la conversión del ěp $\omega \varsigma$ sensible hacia la contemplación de la belleza en sí misma- donde juega un papel importante la reeducación de los deseos, de cara a "purificar el ojo del alma". El ascenso cognoscitivo no es únicamente una cuestión de la inteligencia, sino también del amor, porque el amor es el impulso a la belleza, bien presente en lo sensible, bien en lo inteligible, y sin amor no hay posibilidad de ascenso. Por eso sólo será capaz de contemplar la belleza en sí misma y la idea de bien quien haya primero aprendido mediante el ejercicio práctico a poner orden en los deseos sensibles. Con esto no quiere decir Platón que se anule el deseo sensible (en clave intelectualista), sino que el deseo sensible, purificado, recibe una forma más perfecta y bella. Platón no aboga por una anulación del deseo, sino por una transformación ascética y estética.

En definitiva, el ascenso de las bellezas particulares hacia la belleza en sí misma es un proceso refutatorio apoyado en la fuerza del amor, que busca un objeto satisfactorio para el alma: el amor provoca en el aprendiz la búsqueda de una belleza que le satisfaga, y pronto descubre que los objetos sensibles no colman sus aspiraciones, haciendo que la búsqueda prosiga en la región inteligible ${ }^{42}$. Sin embargo habría que añadir que el ascenso no es únicamente un proceso negativo ${ }^{43}$, sino que también hay una atracción hacia la pureza de las formas que tiene su exponente máximo en la belleza en sí. Es cierto que las bellezas particulares no satisfacen del todo y obligan, al alma del verdadero filósofo, a buscar una belleza más perfecta, pero es igualmente cierto que esas bellezas particulares son el camino, las huellas que indican una belleza superior.

"E $\omega \omega$ s aparece como una fuerza motivacional profunda: el deseo de una belleza plena, total, que colme las aspiraciones del ser humano ${ }^{44}$. Este amor no es $-\mathrm{O}$ no tiene por qué ser- una fuerza ciega, irracional, sino que es susceptible de racionalidad y contribuye de hecho al progreso cognoscitivo. Para Platón el amor es un deseo, pero

41 Kahn, C. "Plato's Theory of Desire". Review of Metaphysics, 41, 1987, p. 101.

42 Sobre el ascenso como proceso refutatorio ver Irwin, T. Plato's Moral Theory. Clarendon Press, Oxford, 1977, p. 171.

43 Cfr. Price, A. W. Love and Friendship in Plato and Aristotle. Clarendon Press, Oxford, 1991, p. 42.

44 Cfr. Pfeil, H. Das platonische Menschenbild. Paul Pattloch, Aschaffenburg, 1963, p. 40. 
un deseo profundo, situado en un plano más interior que el de los apetitos sensibles: las pasiones y deseos son puntuales y concretos, pero el amor no tiene por objeto algo concreto y cambiante, el amor aspira a algo eterno, inmutable, y aspira a llegar más allá de lo meramente sensible ${ }^{45}$. Un amor puro es aquel que se encuentra despojado de la satisfacción sensible inmediata porque apuesta por la contemplación de algo más alto, y por eso mismo alumbra la vida del hombre en todo su recorrido hacia el bien, la belleza y la justicia. Ese amor, correctamente ordenado, no anula los impulsos y las relaciones interpersonales, sino que las transforma y las eleva imprimiendo en ellas la belleza y permitiendo la contemplación. Por eso mismo la contemplación de la verdadera belleza es la que engendra la virtud ${ }^{46}$. El itinerario hacia la belleza no termina en la contemplación, sino que se prolonga en la acción: el deseo de poseer el bien lleva a querer engendrar belleza, tanto en el alma como en tantos otros aspectos de la vida misma ${ }^{47}$. Un planteamiento semejante se encuentra en el Filebo cuando se apunta a que el bien humano consiste en poner límite (orden, racionalidad), a lo ilimitado (los deseos). Es gracias a la contemplación de lo verdaderamente real que el ser humano pone orden en su vida y adquiere la virtud (la justicia, el orden de las partes del alma): en primer lugar porque esa contemplación amorosa le lleva a querer trasladar el orden y la proporción a su fuero interno; en segundo lugar, la contemplación amorosa de lo bueno sólo se puede realizar en la medida en que existe ese orden y proporción.

El concepto de bello ( $\kappa \alpha \lambda o ́ v)$ posee en Platón, al igual que en Aristóteles, connotaciones éticas, estéticas y cognoscitivas. Algo es bello en el campo de lo estético porque responde a unas proporciones armoniosas; un comportamiento es bello y noble porque responde a una estructura ordenada del alma (justicia) ${ }^{48}$. La proporción, el orden y la armonía de las cosas son la belleza que se plasma en la vida, y el amor -como impulso hacia una belleza en sí total y plena- aspira a la contemplación de una armonía perfecta. El amor es el auténtico motor de la virtud ${ }^{49}$.

El amor como deseo es, ciertamente, un intermediario entre el mundo sensible y el inteligible, entre el alma y las ideas, pero es un intermediario importante, un impulso necesario para surcar la senda del verdadero conocimiento y, por lo tanto, algo más que la mera opinión. Esto es así porque el amor no es un deseo más entre otros, sino el deseo más profundo que hay en el ser humano, la fuerza motivacional más

45 Cfr. PIEPER, J. Begeisterung und göttlicher Wahnsinn. Kösel, München, 1962, p. 135.

46 Cfr. Sheffield, F. C. Plato's Symposium. The Ethics of Desire. University Press, Oxford, 2006, pp. 151-153.

47 "From the time that the true lover catches sight of Beauty he enters into a new life in which he is in union with Beauty by means of his appropriate faculty of soul. There is no reason therefore to suppose that his begetting of true virtue is a single and one-off affair; on the contrary, there is every reason to suppose that his knowledge of reality continues to manifest itself in poetry". Whiте, F. C. "Virtue in Plato's Symposium". Classical Quarterly, 54,2004 , p. 373. Un punto curioso de la interpretación de White es que en su opinión la verdadera virtud que engendra la belleza es el producto final de la contemplación (la obra de arte, el discurso filosófico, etc.) y no tanto la virtud interior. Sin embargo, resultaría altamente curioso y sorprendente que para Platón la verdadera virtud se sitúe en el mundo sensible.

48 Cfr. Szalf, J. "Die Aletheia in Platons Tugendlehre". Platon Verstehen. M. van Ackeren (ed.), Wissenschaftlische Buchgesellschaft, Darmstad, 2004, p. 201.

49 En este sentido parece un poco exagerado atribuir a Platón, como hace Nygren, una concepción del amor egocéntrica: "El simple hecho de que el ép $\omega \varsigma$ sea un deseo basta para definirlo como egocéntrico, pues todo deseo es egocéntrico, aunque con distintos grados". Nygren, A. Eros y agape. Trad. J. A. Bravo, Sagitario, Barcelona, 1969, pp. 172-173. El amor es el deseo profundo de plenitud e inmortalidad, equivalente al deseo de felicidad, pero eso no quiere decir que sea egocéntrico, sino que más bien apunta hacia afuera, a las verdades inmutables, en las cuales la persona sale de sí misma en la contemplación de lo más divino y perfecto. 
propiamente humana, que aspira a una belleza en sí o, lo que es lo mismo, a un objeto que lo colme totalmente, y tal satisfacción sólo se lleva a cabo en el terreno de lo inteligible. Al mismo tiempo ese deseo profundo se puede pervertir, puede quedarse atrapado en objetos que no sacian, que únicamente proporcionan una satisfacción sensible, ligada a la غ̇ंı $\theta v \mu$ í $\alpha$. Platón incorpora a su filosofía el amor, no como un elemento instrumental, sino como una fuerza capaz de salvar al hombre o de llevarlo a su propia ruina. De ahí que sea necesaria su propia purificación.

La visión del amor en Platón sería intelectualista si consistiera únicamente en un proceso intelectual que asciende hacia la contemplación de las Formas. Como se ha visto, es posible una interpretación así del Banquete distinta y además, teniendo en cuenta la visión del amor que expone en el Fedro y la República, más bien parece que el ascenso hacia la belleza en sí es un proyecto vital que envuelve deseos, voluntad e inteligencia. La contemplación de la belleza se lleva a cabo mediante una ordenación de los apetitos y la actuación virtuosa. Sólo se asciende y se contempla mejor la belleza en la medida en que nuestra vida es cada vez más bella o, lo que es lo mismo, cuando introducimos orden y armonía: desear de manera bella, actuar de manera bella y, por lo tanto, contemplar cada vez mejor la belleza, hasta reproducir la belleza misma en nuestra vida (pues el amor es deseo de procrear en lo bello, Banquete, 206 e).

\section{Conclusiones}

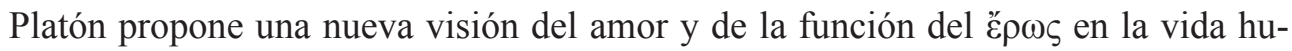
mana. El amor es deseo, pero no un deseo puntual o meramente apetitivo, sino un anhelo hacia la belleza, el bien y la inmortalidad, inscrito en lo más profundo del hombre, y que comienza por la visión de las bellezas particulares para terminar en la contemplación de la belleza en sí misma. Hay amor en la $\dot{\varepsilon} \pi \imath \theta \mu$ í $\alpha$, pero el amor no es $\dot{\varepsilon} \pi \hat{\imath} \theta \mu i_{\alpha} \alpha$, ni tampoco un deseo sensible sublimado hacia el campo artístico o intelectual, porque el amor es anterior y más profundo que los apetitos sensibles. Tampoco es el amor una fuerza cósmica impersonal que mantiene unidas las cosas,

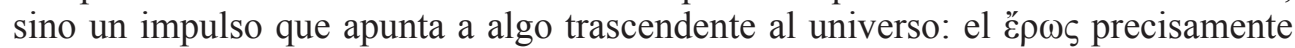
permite al ser humano elevarse por encima del cosmos sensible para penetrar en la belleza del orden inteligible ${ }^{50}$.

De este modo Platón ha conectado el amor y la contemplación, el deseo y el conocimiento, mostrando una imagen del hombre en dinamismo hacia lo eterno mediante el entrenamiento ético y el uso de la inteligencia. El amor es bueno, pero necesita ser purificado y elevado, porque no cualquier objeto - cualquier belleza particularresulta suficiente para colmar las aspiraciones más nobles del ser humano. El alma necesita un alimento adecuado y eso supone elevarse hacia lo inteligible mediante un tipo de vida que modere lo sensible. El amor es impulso hacia la belleza, y a la vez el amor mismo puede ser bello en la medida en que participa del orden y de la armonía de la belleza misma. Por eso Platón no destierra los apetitos y deseos de su filosofía sino que invita a una purificación que ponga orden y medida, del mismo modo que el escultor da forma y gracia a la figura mediante los límites que establece con el cincel. 
El fin de la vida humana en Platón no es únicamente el conocimiento, sino una contemplación práctica, sustentada por un tipo de vida virtuosa y que a su vez permite la transformación y progreso de la realidad. "E $\omega \varsigma$, como anhelo de belleza, es justamente el motor que recorre el camino de ida y vuelta hacia las ideas. El ser humano quiere encontrar la belleza, pero quiere también hacerla suya, encarnarla en la vida misma que vive (la justicia en el alma) y en los objetos con los que se rodea (arte). Por eso podrá afirmar Sócrates que el amor es "de la generación y procreación

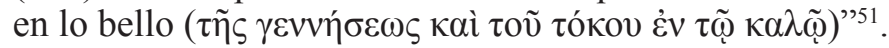

